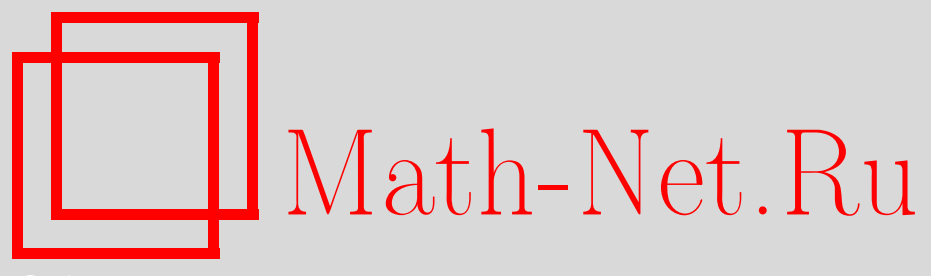

А. А. Зорин, О действии централизатора коизотропной подалгебры в неприводимом представлении простой алгебры Ли, УМН, 2008, том 63, выпуск 6, 167-168

DOI: https://doi.org/10.4213/rm9247

Использование Общероссийского математического портала Math-Net.Ru подразумевает, что вы прочитали и согласны с пользовательским соглашением http://www.mathnet.ru/rus/agreement

Параметры загрузки:

IP : 54.205 .225 .156

26 апреля 2023 г., $17: 28: 56$

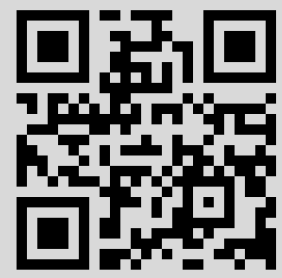




\title{
О действии централизатора коизотропной подалгебры в неприводимом представлении простой алгебры Ли
}

\author{
А. А. Зорин
}

Пусть $\mathfrak{g}=\mathfrak{g}_{n}$ - одна из классических простых комплексных алгебр Ли ранга $n$, т. е. $\mathfrak{s l}_{n+1}(\mathbb{C}), \mathfrak{s o}_{2 n+1}(\mathbb{C}), \mathfrak{s p}_{2 n}(\mathbb{C})$ или $\mathfrak{s o}_{2 n}(\mathbb{C})$ (далее мы будем опускать $\mathbb{C}$ в обозначениях), и $\mathfrak{h} \subset \mathfrak{g}$ - стабилизатор старшего вектора ее стандартного представления.

В [1] доказано, что централизатор $\mathfrak{z} \mathfrak{u}(\mathfrak{g})(\mathfrak{h})$ подалгебры $\mathfrak{h}$ в универсальной обертывающей алгебре $\mathfrak{U}(\mathfrak{g})$ алгебры $\mathfrak{g}$ коммутативен. Более того, $\mathfrak{z} \mathfrak{U}(\mathfrak{g})(\mathfrak{h})$ есть тензорное произведение $\mathfrak{Z}(\mathfrak{g}) \otimes \mathfrak{Z}(\mathfrak{h})$ центров $\mathfrak{Z}(\mathfrak{g})$ и $\mathfrak{Z}(\mathfrak{h})$ алгебр $\mathfrak{U}(\mathfrak{g})$ и $\mathfrak{U}(\mathfrak{h})$ соответственно. $\mathrm{B}$ [1] доказано также, что действие $\mathfrak{h}$ на пуассоновом многообразии $\mathfrak{g}^{*}$ коизотропно. Подалгебры с таким свойством мы называем коизотропными. В [1] доказано, что указанными подалгебрами $\mathfrak{h}$ исчерпывается список коизотропных нередуктивных алгебраических подалгебр с полупростой подалгеброй Леви в простых алгебрах Ли.

Зафиксируем максимальный тор $\mathfrak{t}$ алгебры $\mathfrak{g}$ и систему простых корней $\Pi=$ $\left\{\alpha_{1}, \ldots, \alpha_{n}\right\}$. Имеет место разложение $\mathfrak{g}=\mathfrak{n}_{+} \oplus \mathfrak{t} \oplus \mathfrak{n}_{-}$, где $\mathfrak{n}_{+}$и $\mathfrak{n}_{-}-$унипотентные подалгебры, отвечающие положительным и отрицательным корням соответственно. Положим $\mathfrak{b}_{+}=\mathfrak{n}_{+} \oplus \mathfrak{t}$ и $\mathfrak{b}_{-}=\mathfrak{n}_{-} \oplus \mathfrak{t}$. Через $\omega_{1}$ будем обозначать старший вес стандартного представления алгебры $\mathfrak{g}$, через $\omega$ - минимальный ненулевой вес, пропорциональный весу $\omega_{1}$ и лежащий в решетке корней, а именно, $\omega=n \omega_{1}$ для $\mathfrak{s l}_{n+1}, \omega=\omega_{1}$ для $\mathfrak{s o}_{2 n+1}, \omega=2 \omega_{1}$ для $\mathfrak{s p}_{2 n}$ и $\mathfrak{s o}_{2 n}$. Обозначим через $e_{\alpha}$ корневой вектор, отвечающий корню $\alpha$, а через $\mathfrak{s}$ максимальную редуктивную подалгебру, порожденную элементами $e_{\alpha_{i}}, e_{-\alpha_{i}}$, где $2 \leqslant i \leqslant n$. Алгебра $\mathfrak{s}$ есть классическая простая алгебра Ли $\mathfrak{g}_{n-1}$. Положим $\overline{\mathfrak{t}}=\mathfrak{t} \cap \mathfrak{h}$. Тогда $\overline{\mathfrak{t}}$ есть максимальный тор алгебры $\mathfrak{h}$, выделяемый в $\mathfrak{t}$ уравнением $\omega_{1}(t)=0$. Имеем $\overline{\mathfrak{t}} \subset \mathfrak{s}$ и $\mathfrak{n}_{+} \subset \mathfrak{h}$. Для всякого веса $\lambda \in \mathfrak{t}^{*}$ будем обозначать через $\bar{\lambda}$ его ограничение на $\overline{\mathfrak{t}}$. Если $W$ - некоторый $\mathfrak{t}$-модуль, то через $W_{\mu}$ будем обозначать весовое подпространство веса $\mu \in \mathfrak{t}^{*}$.

Пусть $\rho_{\lambda}: \mathfrak{g} \rightarrow \mathfrak{g l}(V(\lambda))$ - неприводимое представление алгебры Ли $\mathfrak{g}$ с младшим весом $-\lambda$. Его продолжение до представления алгебры $\mathfrak{U}(\mathfrak{g})$ будем также обозначать через $\rho_{\lambda}$. Младший вектор представления $\rho_{\lambda}$ обозначим через $v_{-\lambda}$.

ОПРедЕлЕниЕ. Будем называть вектор $v \in V(\lambda)$ полумладиим, если он является младшим относительно $\mathfrak{s}$ и весовым относительно $\mathfrak{t}$. Линейную оболочку полумладших векторов обозначим через $V(\lambda)^{-}$. Пусть $\bar{\nu} \in \overline{\mathfrak{t}}^{*}$. Множество весов $\lambda$ таких, что $V(\lambda)_{-\bar{\lambda}+\bar{\nu}}^{-} \neq 0$, обозначим через $\mathscr{L}(\bar{\nu})$. Для $\lambda \in \mathscr{L}(\bar{\nu})$ обозначим через $\nu(\lambda) \in \mathfrak{t}^{*}$ минимальный вес такой, что $V(\lambda)_{-\lambda+\nu(\lambda)}^{-} \neq 0$ и $\overline{\nu(\lambda)}=\bar{\nu}$. Веса вида $\nu(\lambda)$ будем называть минимальными. Так же будем называть соответствующие полумладшие векторы.

Нас будет интересовать, как действует алгебра $\mathfrak{z} \mathfrak{u}(\mathfrak{g})(\mathfrak{h})$ в пространстве $V(\lambda)$. Поскольку элементы алгебры $\mathfrak{z} \mathfrak{u}(\mathfrak{g})(\mathfrak{h})$ перестановочны с элементами алгебры $\mathfrak{s}$, то достаточно описать ее действие в пространстве $V(\lambda)^{-}$(ясно, что она сохраняет это подпространство). Так как алгебра $\mathfrak{Z}(\mathfrak{g})$ действует на пространстве $V(\lambda)$ скалярно, то достаточно рассматривать только действие алгебры $\mathfrak{Z}(\mathfrak{h})$. Обозначим через $\mathfrak{Z}(\mathfrak{h})_{+}$ идеал в $\mathfrak{Z}(\mathfrak{h})$, состоящий из элементов с нулевым свободным членом. Основной гипотезой о действии $\mathfrak{Z}(\mathfrak{h})$ в пространстве $V(\lambda)$ является следующая

ГиПотезА. Пусть $\mathfrak{g}-$ классическая простая алгебра Ли, $\mathfrak{h}$ - стабилизатор старшего вектора тавтологического представления алгебры $\mathfrak{g}$. Пусть $\rho_{\lambda}: \mathfrak{g} \rightarrow \mathfrak{g l}(V)-$ неприводимое представление с младшим весом - $\lambda$. Тогда спектр $\mathfrak{s - м о д у л я ~} W(\lambda)=$ $V(\lambda) / \rho_{\lambda}\left(\mathfrak{Z}(\mathfrak{h})_{+}\right) V(\lambda)$ nрост.

Утверждение гипотезы означает следующее: существует не более одного минимального полумладшего вектора с заданной проекцией на $\mathfrak{t}^{*}$, и если $v_{-\lambda+\nu}-$ минимальный полумладший вектор, то выполняется равенство $V(\lambda)_{-\bar{\lambda}+\bar{\nu}}^{-}=\mathfrak{Z}(\mathfrak{h}) v_{-\lambda+\nu}$.

Строение алгебры $\mathfrak{Z}(\mathfrak{h})$ описывается следующей теоремой. 
Tеорема 1 [1]. Алгебра $\mathfrak{Z}(\mathfrak{h})$ есть алгебра многочленов $\mathbb{C}\left[F_{1}, \ldots, F_{d}\right]$. При этом $d=1 \partial \Omega я \mathfrak{g}=\mathfrak{s l}_{n+1}, d=n \partial \Omega я \mathfrak{g}=\mathfrak{s p}_{2 n} u \mathfrak{g}=\mathfrak{s o}_{2 n+1}, d=n-1$ для $\mathfrak{g}=\mathfrak{s o}_{2 n}$. Более того,

1) в случаях $\mathfrak{g}=\mathfrak{s l}_{n+1}, \mathfrak{s o}_{2 n}, \mathfrak{s p}_{2 n}$ можно выбрать порождающие $F_{1}, \ldots, F_{d}$ так, что они имеют вес $\omega$ относительно максимального тора $\mathfrak{t}$;

2) в случае $\mathfrak{g}=\mathfrak{s o}_{2 n+1}$ можно выбрать порождающие $F_{1}, \ldots, F_{n}$ так, что многочлен $F_{1}$ имеет вес $\omega$, а многочлены $F_{2}, \ldots, F_{n}$ - вес $2 \omega$ относительно $\mathfrak{t}$.

Обозначим через $\mathfrak{Z}(\mathfrak{h})_{p}$ линейную оболочку элементов из $\mathfrak{Z}(\mathfrak{h})$, имеющих вес $p \omega$ относительно $\mathfrak{t}$. Подпространства $\mathfrak{Z}(\mathfrak{h})_{p \omega}, p=0,1,2, \ldots$, задают $\mathbb{Z}$-градуировку на $\mathfrak{Z}(\mathfrak{h})$. Положим $\left\langle\lambda, \alpha_{i}\right\rangle=2\left(\lambda, \alpha_{i}\right) /\left(\alpha_{i}, \alpha_{i}\right)$. В данной работе мы докажем следующий асимптотический вариант сформулированной выше гипотезы.

Теорема 2. Для любого минимального полумладшего веса $\nu$ и фиксированного целого р существуют такие числа $K_{1}(\nu), \ldots, K_{n}(\nu)$, что для всех $\lambda$ таких, что $\left\langle\lambda, \alpha_{i}\right\rangle \geqslant K_{i}(\nu)$, выполняются следующие два условия:

1) $\mathfrak{Z}(\mathfrak{h})_{p \omega} v_{-\lambda+\nu}=V(\lambda)_{-\lambda+\nu+p \omega}^{-}$;

2) $\operatorname{dim} \mathfrak{Z}(\mathfrak{h})_{p \omega} v_{-\lambda+\nu}=\operatorname{dim} V(\lambda)_{-\lambda+\nu+p \omega}^{-}=\operatorname{dim} \mathfrak{Z}(\mathfrak{h})_{p \omega}$.

Нам потребуются две леммы, которые мы приведем без доказательства.

Обозначим через $J(\lambda) \subset \mathfrak{U}(\mathfrak{g})$ стабилизатор вектора $v_{-\lambda}$ в $V(\lambda)$. Известно, что $J(\lambda)=I(\lambda) \oplus N(\lambda)$, где $I(\lambda)=\mathfrak{U}(\mathfrak{g}) \mathfrak{n}_{-}+\sum_{h \in \mathfrak{t}} \mathfrak{U}\left(\mathfrak{b}_{+}\right)(h+\lambda(h)) \subset \mathfrak{U}(\mathfrak{g})$ и $N(\lambda) \subset \mathfrak{U}\left(\mathfrak{n}_{+}\right)-$ левый идеал, порожденный элементами $e_{\alpha_{i}}^{a_{i}+1}$, где $a_{i}=\left\langle\lambda, \alpha_{i}\right\rangle$ (см. [2]).

Лемма 1. 1) Для любого веса $\lambda$ и любого р верно равенство $\mathfrak{Z}(\mathfrak{h})_{p \omega} \cap I(\lambda)=0$.

2) Для любого фиксированного веса $\mu \in \mathfrak{t}^{*}$ существуют такие числа $K_{1}(\mu), \ldots$, $K_{n}(\mu)$, что для всех $\lambda$ таких, что $\left\langle\lambda, \alpha_{i}\right\rangle \geqslant K_{i}(\mu)$, и всех $\eta$ таких, что вес $\mu-\eta$ является суммой простых корней с целыми коэффициентами, выполняется равенство $\mathfrak{U}\left(\mathfrak{n}_{+}\right)_{\mu} \cap J(\lambda)=0$.

Лемма 2. 1) для любого веса $\lambda \in \mathscr{L}(\bar{\nu}), \quad \bar{\nu} \in \overline{\mathfrak{t}}^{*}$, имеет место неравенство $\left.\operatorname{dim} V(\lambda)_{-\lambda+\nu(\lambda)+p \omega}^{-} \leqslant \operatorname{dim} \mathfrak{Z}(\mathfrak{h})_{p \omega} ; 2\right)$ вес $\nu(\lambda)$ не зависит от $\lambda \in \mathscr{L}(\bar{\nu}), \bar{\nu} \in \overline{\mathfrak{t}}^{*}$.

Из работ [3]-[5] можно явным образом получить число полумладших векторов фиксированного веса и убедиться, что оно удовлетворяет условию 1) леммы 2. Также из результатов [3]-[5] следует, что минимальный вес не зависит от $\lambda$.

КРАТКОЕ ДОКАЗАТЕЛЬСТВО ТЕОРЕМЫ 2. Зафиксируем минимальнЫЙ полумЛадший вес $\nu$ и вес $p \omega$, обозначим $\mu=\nu+p \omega$. Положим $K_{i}(\nu)=K_{i}(\mu)$, где $K_{i}(\mu)$ - числа из леммы 1 для веса $\mu$. Пусть $V(\lambda)$ - неприводимый $\mathfrak{g}$-модуль и $\left\langle\lambda, \alpha_{i}\right\rangle \geqslant K_{i}(\nu)$. Тогда $\mathfrak{U}\left(\mathfrak{n}_{+}\right)_{p \omega} \cap N(\lambda)=0$ и, следовательно, $\mathfrak{Z}(\mathfrak{h})_{p \omega} \cap J(\lambda)=\mathfrak{Z}(\mathfrak{h})_{p \omega} \cap I(\lambda)=0$ (лемма 1$)$.

Пусть $c \in \mathfrak{Z}(\mathfrak{h})_{p \omega}, c \neq 0$ и $v_{-\lambda+\nu}=u_{\nu} v_{-\lambda}-$ минимальный полумладший вектор, $u_{\nu} \in \mathfrak{U}\left(\mathfrak{n}_{+}\right)_{\nu}$. Имеем $c u_{\nu} v_{-\lambda}=u_{\nu} c v_{-\lambda}$, и выполнено $c v_{-\lambda}=u_{0} v_{-\lambda} \neq 0$ для некоторого $u_{0} \in \mathfrak{U}\left(\mathfrak{n}_{+}\right)_{p \omega}$. Так как $u_{\nu} u_{0} \in \mathfrak{U}\left(\mathfrak{n}_{+}\right)_{\mu}$, то $u_{\nu} u_{0} v_{-\lambda} \neq 0$ (лемма 1 ) и, следовательно, $c v_{-\lambda+\nu} \neq 0$. Таким образом, $\operatorname{dim} \mathfrak{Z}(\mathfrak{h})_{p \omega} v_{-\lambda+\nu}=\operatorname{dim} \mathfrak{Z}(\mathfrak{h})_{p \omega}$. Из леммы 2 следует, что $V(\lambda)_{-\lambda+\nu+p \omega}^{-} \leqslant \operatorname{dim} \mathfrak{Z}(\mathfrak{h})_{p \omega}$. Поскольку $\mathfrak{Z}(\mathfrak{h})_{p \omega} v_{-\lambda+\nu} \subset V(\lambda)_{-\lambda+\nu+p \omega}^{-}$и размерность левой части не меньше размерности правой, то включение превращается в равенство.

\section{Список литературы}

[1] А. А. Зорин, "О коммутативности централизатора подалгебры в универсальной обертывающей алгебре", Функи. анализ и его прил. (в печати). [2] Ж. Диксмье, Универсалъные обертывающие алгебры, Мир, М., 1978. [3] В. В. Штепин, Изв. РАН. Сер. матем., 57:6 (1993), 176-198. [4] В. В. Штепин, Изв. РАН. Сер. матем., 62:3 (1998), 201-223. [5] В. В. Штепин, Изв. РАН. Сер. матем., 68:2 (2004), 159-190.

\section{А. А. Зорин (А. А. Zorin)}

Московский государственный университет им. М. В. Ломоносова

E-mail: aa-zorin@yandex.ru
Представлено Э. Б. Винбергом Принято редколлегией 04.10 .2008 